\title{
Quantum treatment of phonon scattering for three-dimensional atomistic transport modeling
}

\author{
Y. Lee ${ }^{1, *}$ M. Bescond,${ }^{1}{ }^{\dagger}$ N. Cavassilas,${ }^{1}$ D. Logoteta,${ }^{1}$ L. Raymond, ${ }^{1}$ M. Lannoo, ${ }^{1}$ and M. Luisier ${ }^{2}$ \\ ${ }^{1}$ IM2NP, UMR CNRS 7334, Aix-Marseille Université, Technopôle de Château-Gombert, \\ Bâtiment Néel, 60 Rue Frédéric Joliot Curie, 13453 Marseille, France \\ ${ }^{2}$ Integrated Systems Laboratory, ETH Zürich, 8092 Zürich, Switzerland
}

\begin{abstract}
Based on the non-equilibrium Green's function (NEGF) formalism, we show a numerically efficient method to treat inelastic scattering in multi-dimensional atomistic codes. Using a simple rescaling approach, we detail the calculations of the lowest order approximation (LOA) ${ }^{1}$ series to the usual, computationally intensive, self-consistent Born approximation (SCBA). This, combined with the analytic continuation technique of Padé approximants, is applied to an atomistic code based on a tight-binding $s p^{3} d^{5} s^{*}$ model for electrons and holes, and a modified valence-force-field method for phonons. Currents in $\mathrm{Si}$ and Ge gate-all-around nanowire transistors are then computed considering the main crystallographic transport directions $(\langle 100\rangle,\langle 110\rangle,\langle 111\rangle)$ for both n-type and p-type devices. Our results show that in most configurations, $3^{\text {rd }}$ order LOA currents are enough to achieve a high agreement with SCBA results, while reducing the calculation time by about one order. In addition, we propose a criterion to determine the validity of such expansion techniques.
\end{abstract}

The recent introduction of three-dimensional (3D) Fin structured tri-gate transistors in the semiconductor industry has opened the door for a steady continuation of Moore's scaling law in the sub-20 nm technology node ${ }^{2}$. However, despite the success of the device architecture for the mass production, there is a remaining question: Will this device structure be still valid for transistors with sub-10 $\mathrm{nm}$ gate lengths? In this perspective, the ongoing researches mainly focus on $i$ ) improving the electrostatic control of the device architecture like gate-allaround (GAA) Si nanowire ${ }^{3}$, or $i$ ) searching novel materials exhibiting high mobilities and/or high band-gap ${ }^{4-9}$, or iii) the combination of both aspects, like Wrap-Gated (WG) InGaAs III-V nanowire transistors ${ }^{10-12}$.

In all the aforementioned devices both theoretical ${ }^{13-16}$ and experimental ${ }^{17}$ works underline the impact of the position of each individual atom on the obtained performance. Indeed, physics of nano-scaled devices is mainly governed by quantum effects (confinement ${ }^{18}$ and tunneling ${ }^{19,20}$ ), surface roughness scattering ${ }^{21}$ and electron-phonon interactions ${ }^{22}$. Among those phenomena, it has been theoretically predicted ${ }^{9,23}$ that inelastic interactions between electrons and phonons play a major role even in ultra scaled devices with dimensions of the order of the electron mean free path. Thus, in order to analyze the physical properties of the devices, the development of atomistic quantum simulation tools including inelastic electron-phonon scattering is urgently needed. During recent decades, several quantum methods have been proposed to treat this issue precisely. Among them the non-equilibrium Green's function (NEGF) formalism ${ }^{24-26}$ is one of the most advanced quantum methods that has attracted intensive interests due to its suitability for addressing inelastic carrier transport in nanostructures.

Unfortunately, atomistic NEGF quantum transport simulations of realistic devices require important numerical resources. The situation becomes even more complicated when inelastic interactions are included. In- deed phonon scattering is usually treated with the selfconsistent Born approximation (SCBA) that induces an additional self-consistent scheme to the conventional "Poisson-Schrödinger" loop. The SCBA was first applied to simple effective mass or $\mathrm{k} \cdot \mathrm{p}$ Hamiltonians ${ }^{27,28}$. It was then implemented in 3D full-band atomistic NEGF codes based on tight-binding Hamiltonian ${ }^{22,29}$. Among those advanced codes, the simulator OMEN developed by Luisier and co-workers is one of the most sophisticated that couples inelastic transport of phonons and electrons with an atomistic precision ${ }^{30,31}$. However, this simulator is only manageable with more than $1000 \mathrm{CPUs}$ due to unavoidable numerical burden.

Recently we proposed a technique that allows to highly reduce the computational complexity for taking into account the interaction between electrons and phonons within the NEGF framework. The approach ${ }^{1,32,33}$ is based on a lowest order approximation (LOA) of the interactions combined with an analytic continuation technique of Padé approximants ${ }^{34,35}$. Its main advantage is to avoid the numerous iterations required by SCBA. However, it implies the inversions and multiplications of potentially large matrices which may be impractical for $3 \mathrm{D}$ systems. We here present a simple rescaling technique able to calculate a series of LOA using the first iterations of the conventional SCBA algorithm. We apply this efficient technique to the atomistic code $\mathrm{OMEN}^{30}$ to model 3D nanowire (NW) transistors for which electronphonon scattering is important. In particular, we show that the physical quantities (i.e. the currents) based on the $3^{\text {rd }}$ order LOA combined with Padé analytic continuation technique are enough to obtain the results of the full SCBA iterations. Our investigations focus on both ntype and p-type Si GAA NW considering the three main crystallographic orientations $(\langle 100\rangle,\langle 110\rangle,\langle 111\rangle)$. Application to Ge NW in $\langle 110\rangle$ transport direction is also discussed.

The device structure investigated in this paper is schematized in Fig. 1. The chosen gate length is $5 \mathrm{~nm}$ 


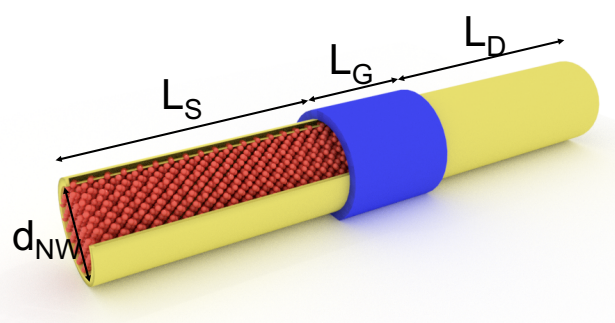

FIG. 1. (Color online) Schematic representation of the silicon GAA NW transistors considered in this work (with a $\langle 100\rangle$ crystallographic orientation in the present case). The length of the gate is $L_{G}=5 \mathrm{~nm}$, the source and drain extensions $L_{S / D}=15 \mathrm{~nm}$, and the diameter of NW $d_{N W}=3 \mathrm{~nm}$. Source and drain regions are doped with a doping concentration of donors or acceptors $N_{D / A}=1 \times 10^{20} \mathrm{~cm}^{-3}$. Si $\langle 110\rangle$, $\mathrm{Si}\langle 111\rangle$, and $\mathrm{Ge}\langle 110\rangle$ are also considered (not shown).

since it is considered as an ultimate limit for conventional logic transistors. The channel is surrounded by a $3 \mathrm{~nm}$ thick $\mathrm{HfO}_{2}$ dielectric layer (with $\epsilon_{R}=20$ ), while source and drain regions are covered by low $\kappa$ dielectric layer (with $\epsilon_{R}=5$ ). A supply voltage $\left|V_{D S}\right|=\left|V_{D D}\right|=$ $0.6 \mathrm{~V}$ is applied between the source and drain contacts, and a gate-to-source bias $V_{G S}$ then controls the currents flowing inside the channel. Since electron-phonon scattering is found to be stronger in the saturation regime ${ }^{22}$, we focus here on this operation mode.

We consider steady-state electron transport described within the NEGF framework employing a full band tightbinding $s p^{3} d^{5} s^{*}$ model without spin-orbit coupling ${ }^{36,37}$. The lateral surface of the semiconductor NW in which we solve the Schrödinger's equation is passivated by increasing the energy of dangling bonds ${ }^{38}$, and no surface roughness scattering is considered. Phonon relation dispersion in the confined NW is obtained via a modified valenceforce-field method including four interaction terms ${ }^{39,40}$. Here, as described in Ref. 30, electrons are coupled to phonons through the following diagonal lesser or greater self-energy

$$
\begin{aligned}
\Sigma_{n n}^{\lessgtr \sigma_{1} \sigma_{2}}(E)= & \mathrm{i} \sum_{l} \sum_{i j} \sum_{\sigma_{3} \sigma_{4}} \int_{-\infty}^{\infty} \frac{\mathrm{d}(\hbar w)}{2 \pi} \nabla_{i} H_{n l}^{\sigma_{1} \sigma_{3}} \\
& \times G_{l l}^{\lessgtr \sigma_{3} \sigma_{4}}(E \mp \hbar \omega) \nabla_{j} H_{l n}^{\sigma_{4} \sigma_{2}}\left(D_{l n}^{\lessgtr i j}(\omega)\right. \\
& \left.-D_{l l}^{\lessgtr i j}(\omega)-D_{n n}^{\lessgtr i j}(\omega)+D_{n l}^{\lessgtr i j}(\omega)\right),
\end{aligned}
$$

where $\hbar$ is the reduced Planck's constant, $E$ the electron energy, $\omega$ the phonon frequency, $H_{n l}$ the Hamiltonian (tight-binding matrix element), $G(E)$ the electron Green's function, and $D(\omega)$ the phonon Green's function expressed in the real-space (the indices $i$ and $j$ run over $x, y$, and $z$ directions). The other indices $n$ and $l$ denote all atomic positions, and $\sigma$ represent the orbitals. $\nabla_{i} H_{n l}$ represents the first derivative of the tight-binding Hamiltonian matrix $H_{n l}$ along the $i$ direction $(x, y$, or $z)$. It defines the electron-phonon coupling due to atom oscillations around their equilibrium position (please see Ref.[30] for more details). We assume a bath of phonons at thermal equilibrium by considering no temperature gradients inside the device and by neglecting the phonon self-energies which can contribute to the phonon Green's function $D(\omega)$ (see Ref. 30). We should note that the diagonal approximation of the self-energy, which results from computational limitations in atomistic codes, may lead to an underestimation of the strength of electronphonon coupling ${ }^{41,42}$. However, Ref. 43 showed that the diagonal approximation was still valid in ultra-scaled $\mathrm{Si}$ nanowires for electrons, except for transport along the $\langle 110\rangle$ crystal orientation. More generally, the LOA combined with Padé approximant can be applied to nondiagonal self-energies. The self-energy of Eq.(1) combined with Dyson's equation ${ }^{44}$ allows to calculate the electron interacting Green's function $G(E)$ starting from the non-interacting one $g(E)$.

$$
G=g+g \Sigma[G] G,
$$

where the simplified matrix notation $\left[1=\left(r_{1}, t_{1}\right)\right.$, $\left.g \Sigma[G] G=\int d 2 \int d 2^{\prime} g(1 ; 2) \Sigma\left(2 ; 2^{\prime}\right) G\left(2^{\prime} ; 1^{\prime}\right)\right]$ is used. Dyson's equation (2) is typically solved using the socalled iterative SCBA scheme with a specified convergence tolerance such as

$$
G_{N}=\left[g^{-1}-\Sigma\left[G_{N-1}\right]\right]^{-1}
$$

where $G_{N}$ is the Green's function at the $N^{t h}$ SCBA iteration and $G_{0}=g$.

Green's functions resulting from first few iterations (i.e. $\quad N$ small in Eq.(3)) of the SCBA scheme are generally not conserving for strong electron-phonon interactions $^{1,33}$. The SCBA might then require more than several dozens of iterations to provide accurate conserving physical properties. On the other hand, LOA Green's functions are conserving at any order. It has been shown that the first few orders of LOA Green's functions coupled with Padé approximants can highly accelerate the calculations of physical quantities (both current and charge density) ${ }^{1}$. However, the LOA Green's function algorithm presented in Ref. 1 implies numerous inversions and multiplications of Green's function matrices. Its implementation is then numerically very demanding when applied to 3D nanostructures. Here, we show that the derivation of LOA Green's functions from the usual SCBA algorithm can be easily reached by introducing a scaling parameter to Eq.(1).

As an example, let us rewrite Eq.(3) in the Taylor series expansion as

$$
\begin{aligned}
G_{N} & =g+g \Sigma\left[G_{N-1}\right] g \\
& +g \Sigma\left[G_{N-1}\right] g \Sigma\left[G_{N-1}\right] g+\cdots .
\end{aligned}
$$

The first SCBA Green's function $G_{1}$ is then defined from Eq.(4) as

$$
G_{1}=g+g \Sigma[g] g+g \Sigma[g] g \Sigma[g] g+\cdots .
$$




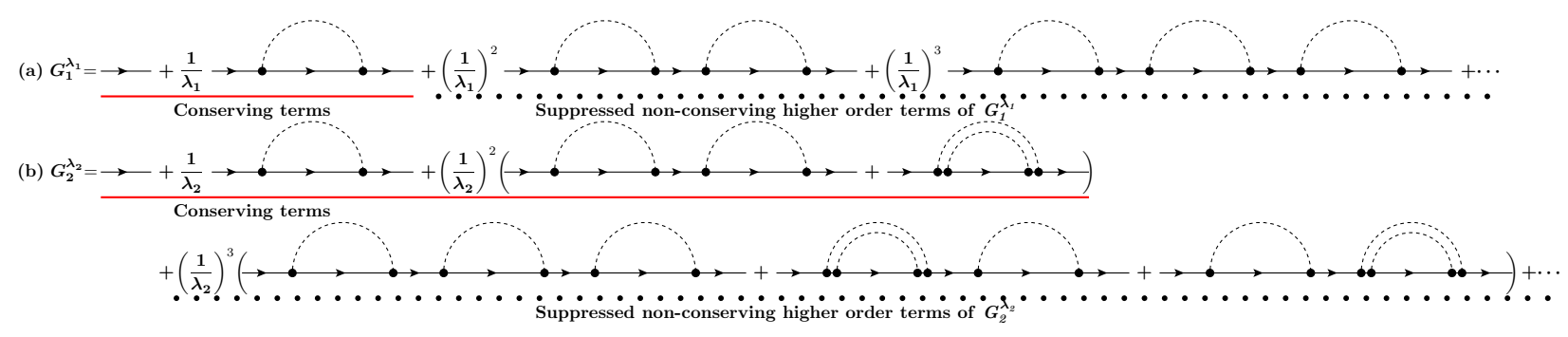

FIG. 2. (Color online) Rescaled Green's functions, (a) $G_{1}^{\lambda_{1}}$ of first SCBA iteration and (b) $G_{2}^{\lambda_{2}}$ of second SCBA iteration, described by Feynman diagrams. Conserving and non-conserving terms are arranged in ascending order in interaction. Thin lines with an arrow indicate electron non-interacting Green's function. Dashed lines are for free phonon propagators.

$G_{1}$ is not conserving due to second and higher order terms in interaction ${ }^{33}$. Its first order LOA counterpart $G_{\text {LOA1 }}$ is conserving and expressed as

$$
G_{L O A 1}=g+g \Sigma[g] g .
$$

$G_{L O A 1}$ can then be deduced from $G_{1}$ by introducing a scaling parameter $1 / \lambda_{1}$ in the self-energy of Eq.(1). $G_{1}$ rescaled by $\lambda_{1}$ is now defined by:

$$
G_{1}^{\lambda_{1}}=g+\frac{1}{\lambda_{1}} g \Sigma[g] g+\left(\frac{1}{\lambda_{1}}\right)^{2} g \Sigma[g] g \Sigma[g] g+\cdots .
$$

Taking a relevant value of $\lambda_{1}$, the factors $\left(1 / \lambda_{1}\right)^{n}(n>1)$ can eliminate the infinite sum of non-conserving terms while preserving the first order conserving one. In NEGF, since any relevant expectation value $(\mathcal{O})$ is linear in the one-electron Green's function, the expectation value of the first order term to $G_{\mathrm{LOA} 1}$ can be constructed as $\Delta \mathcal{O}_{1}=\mathcal{O}(g \Sigma[g] g)=\lambda_{1}\left[\mathcal{O}\left(G_{1}^{\lambda_{1}}\right)-\mathcal{O}(g)\right]$.

The same technique can be applied to the second SCBA iteration of Eq.(4) considering a different scaling factor $\lambda_{2}$ to obtain the rescaled $G_{2}$ as

$$
\begin{aligned}
G_{2}^{\lambda_{2}} & =g+\frac{1}{\lambda_{2}} g \Sigma\left[G_{1}^{\lambda_{2}}\right] g \\
& +\left(\frac{1}{\lambda_{2}}\right)^{2} g \Sigma\left[G_{1}^{\lambda_{2}}\right] g \Sigma\left[G_{1}^{\lambda_{2}}\right] g+\cdots,
\end{aligned}
$$

where $G_{1}^{\lambda_{2}}$ is $G_{1}$ rescaled by $\lambda_{2}$. Since the SCBA selfenergy is linear with respect to the Green's function ${ }^{33}$, the rescaled $G_{2}$ can be expressed as

$$
\begin{aligned}
G_{2}^{\lambda_{2}} & =g+\frac{1}{\lambda_{2}} g \Sigma[g] g \\
& +\left(\frac{1}{\lambda_{2}}\right)^{2}(g \Sigma[g] g \Sigma[g] g+g \Sigma[g \Sigma[g] g] g) \\
& +\left(\frac{1}{\lambda_{2}}\right)^{3}(g \Sigma[g] g \Sigma[g] g \Sigma[g] g+\cdots)+\cdots
\end{aligned}
$$

where $\lambda_{2}$ should be chosen to remove higher order terms associated to $\left(1 / \lambda_{2}\right)^{n}(n>2)$, but to maintain the first and second order conserving terms. With this scaling parameter, the expectation value of the second order terms to second order LOA Green's function $G_{L O A 2}$ can be obtained as $\Delta \mathcal{O}_{2}=\mathcal{O}(g \Sigma[g] g \Sigma[g] g+g \Sigma[g \Sigma[g] g] g)=$ $\lambda_{2}^{2}\left[\mathcal{O}\left(G_{2}^{\lambda_{2}}\right)-\mathcal{O}(g)\right]-\lambda_{2} \Delta \mathcal{O}_{1}$. Figures 2 (a) and $2(\mathrm{~b})$ illustrate this scaling technique in terms of Feynman diagrams for the first and second SCBA iterations, respectively.

Once the rescaled conserving Green's functions at each order are obtained, the expectation values of the desired observable can be reconstructed using the linearity property of the corresponding operator. In particular, $N^{t h}$ order LOA current can be expressed as

$$
\mathcal{I}_{N^{t h} L O A}=\mathcal{I}_{0}+\sum_{n=1}^{N} \triangle \mathcal{I}_{n},
$$

with

$$
\begin{aligned}
\triangle \mathcal{I}_{N}= & \left(\lambda_{N}\right)^{N} \mathcal{I}_{N^{t h} S C B A}\left(G_{N}^{\lambda_{N}}\right) \\
& -\sum_{n=0}^{N-1}\left(\lambda_{N}\right)^{N-n} \triangle \mathcal{I}_{n}, \quad \triangle \mathcal{I}_{0}=\mathcal{I}_{0} .
\end{aligned}
$$

This procedure demonstrates that conserving Green's function at any order $N$ can be in principle obtained from conventional SCBA scheme. At each order $N, \lambda_{N}$ has to be large enough to remove all non-conserving terms (whose order in interaction is larger than $N$ ) but small enough to maintain conserving terms (whose order in interaction is $\leq N)$. This approach relies on the relevant choice of $\lambda_{N}$ which can be rapidly verified through the current conservation. Explicitly we determine the $\lambda_{N}$ 's based on a semi-empirical method noting that they are associated to a Taylor development of the Green's function. We first find the scaling parameter $\lambda_{1}$, which ensures that the current is conserved after the first SCBA iteration. We then deduce an approximated value of $\lambda_{2}$ as $2 \times\left(\lambda_{1}\right)^{1 / 2}$. Based on the same argument, we take $\lambda_{3}$ equal to $5 \times\left(\lambda_{1}\right)^{1 / 3}$. The multiplication factors 2 and 5 for $\lambda_{2}$ and $\lambda_{3}$, respectively represent the weighting according to the number of diagrams at each order. We 
TABLE I. Comparisons of 3 first LOA currents and corresponding Padé approximants $(0 / 1,1 / 1$, and 1/2) with the ballistic and SCBA currents in $\mathrm{n}-\mathrm{Si}\langle 100\rangle, \mathrm{n}-\mathrm{Si}\langle 110\rangle, \mathrm{n}-\mathrm{Si}\langle 111\rangle$, and $\mathrm{n}-\mathrm{Ge}\langle 110\rangle$ devices. Relative differences $(\varepsilon)$ of the LOA and Padé currents with respect to the SCBA values are also reported $\left(\varepsilon=100 \times\left|\mathcal{I}_{S C B A}-\mathcal{I}\right| / \mathcal{I}_{S C B A}\right)$ where $\mathcal{I}$ is ballistic, LOA, or Padé current.

\begin{tabular}{cccccccccc}
\hline \hline & Ballistic & LOA1 & LOA2 & LOA3 & Padé 0/1 & Padé 1/1 & Padé 1/2 & SCBA & Device \\
\hline Current [A] & $8.12 \mathrm{e}-6$ & $6.40 \mathrm{e}-6$ & $-2.37 \mathrm{e}-5$ & $2.47 \mathrm{e}-4$ & $6.70 \mathrm{e}-6$ & $8.22 \mathrm{e}-6$ & $5.05 \mathrm{e}-6$ & $5.26 \mathrm{e}-6$ & $\mathrm{n}-\mathrm{Si}\langle 100\rangle$ \\
$\varepsilon[\%]$ & 54.4 & 21.7 & 550.6 & 4595.8 & 27.4 & 56.3 & 4.0 & 0.0 \\
\hline Current [A] & $6.55 \mathrm{e}-6$ & $4.58 \mathrm{e}-6$ & $-3.92 \mathrm{e}-5$ & $1.61 \mathrm{e}-3$ & $5.04 \mathrm{e}-6$ & $6.64 \mathrm{e}-6$ & $4.42 \mathrm{e}-6$ & $4.45 \mathrm{e}-6$ & $\mathrm{n}-\mathrm{Si}\langle 110\rangle$ \\
$\varepsilon[\%]$ & 47.2 & 2.9 & 980.9 & 36079.8 & 13.3 & 49.2 & 0.7 & 0.0 \\
\hline Current [A] & $4.34 \mathrm{e}-6$ & $1.72 \mathrm{e}-5$ & $-4.38 \mathrm{e}-5$ & $2.03 \mathrm{e}-4$ & $-2.20 \mathrm{e}-6$ & $6.59 \mathrm{e}-6$ & $5.48 \mathrm{e}-6$ & $6.01 \mathrm{e}-6$ & $\mathrm{n}-\mathrm{Si}\langle 111\rangle$ \\
$\varepsilon[\%]$ & 27.8 & 186.2 & 828.8 & 3277.7 & 136.6 & 9.6 & 8.8 & 0.0 \\
\hline Current [A] & $5.27 \mathrm{e}-6$ & $4.76 \mathrm{e}-6$ & $2.09 \mathrm{e}-6$ & $7.11 \mathrm{e}-5$ & $4.80 \mathrm{e}-6$ & $5.39 \mathrm{e}-6$ & $4.72 \mathrm{e}-6$ & $4.62 \mathrm{e}-6$ & $\mathrm{n}-6 \mathrm{Ge}\langle 110\rangle$ \\
$\varepsilon[\%]$ & 14.1 & 3.0 & 54.8 & 1439.0 & 3.9 & 16.7 & 2.2 & 0.0 \\
\hline \hline
\end{tabular}

are aware of the empirical character of this approach, but the assessment of scaling parameters at any order in interactions and to any systems is a very complex issue beyond the scope of the present work. Here, we calculate up to $3^{\text {rd }}$ order currents and apply Padé approximants $0 / 1,1 / 1$, and $1 / 2$. Calculation of higher order LOA currents is also possible, but it usually requires additional SCBA iterations that could reduce the relevance of the presented technique. Moreover, the approach could have been also applied to the calculation of the charge carrier density by using the same scaling parameters as those for the current. For the sake of clarity, we decided to only focus on the current considerations. The comparisons between SCBA and LOA/Padé approximant are performed with the same electrostatic potential profile as the one resulting from the self-consistent loop between the Poisson equation and the NEGF formalism including the interactions within the SCBA scheme.

Table I shows comparisons of the first three LOA orders and corresponding Padé currents with SCBA values for n-Si (n-type silicon) and n-Ge (n-type germanium) devices along several principal crystallographic orientations. We first see that all LOA current series (LOA1, LOA2, and LOA3) are diverging from SCBA currents, meaning that the considered devices operate in a relatively strong electron-phonon interaction regime ${ }^{1}$. Padé 0/1 applied to the first order LOA currents shows an underestimation of the current degradation compared with the SCBA currents, except for $\mathrm{n}$-Si $\langle 111\rangle$-oriented device. In addition, Padé $1 / 1$ approximants based on up to $2^{\text {nd }}$ order LOA currents are in most cases not reliable as pointed out in previous work ${ }^{1}$. However, the application of Padé $1 / 2$ on LOA current series shows significant improvements, with high accuracies compared with the SCBA values. For $\mathrm{n}-\mathrm{Si}$ and $\mathrm{n}-\mathrm{Ge}\langle 110\rangle$-oriented devices, Padé $1 / 2$ provides almost the same values as the SCBA currents with a relative difference $(\varepsilon)$ of less than $2 \%$ on average. It is also worth noting that Padé $1 / 2$ can provide a reasonably good result $(<9 \%$ relative difference) for $\langle 111\rangle$ transport direction where SCBA needs more than 40 iterations to converge. Therefore, currents resulting from Padé $1 / 2$ can be seen as a good estima- tion to the SCBA values with a relative difference less than $10 \%$ for all the considered n-type devices. These results confirm the previous work ${ }^{1}$ where it has been shown that Padé $N / N+1$ can give better results even in strong electron-phonon interacting system.

Table II shows similar results as Table I for p-type devices. We see that all LOA currents (LOA1, LOA2, and LOA3) are divergent series like in the case of n-type devices. Table II also reports very favorable results for the $\langle 110\rangle$ direction for which even the simplest Padé $0 / 1$ guarantees a high agreement of the current $(\varepsilon<6 \%)$ with respect to the SCBA approach. Padé $1 / 1$ also produces reliable results for the $\langle 110\rangle$ direction while remaining unstable for the other configurations. Padé $1 / 2$ still gives current value very close to those of SCBA with $\varepsilon$ less than $6 \%$. The result is also remarkable for the $\langle 100\rangle$ direction where Padé $1 / 2$ provides similar accuracy while SCBA requires more than 80 iterations. However, the comparison is less successful for the $\mathrm{p}-\mathrm{Si}\langle 111\rangle$ configuration for which all LOA and Padé currents fail to reproduce the SCBA result.

The transport along the $\langle 111\rangle$ direction is quite singular for both n-type and p-type devices. For that orientation first order LOA currents are larger than the ballistic values (see Tables I and II). Concerning the n-type device the converged SCBA current is also larger than the ballistic one and the Padé $1 / 2$ finally succeeds to provide an accurate value. In n-type $\langle 111\rangle$-oriented NW the counterintuitive increase of the current in the presence of phonon scattering with respect to the ballistic value results from the interplay between two competing effects: first, backscattering caused by phonon emission or absorption tends to decrease the current magnitude. Secondly, inter-subband scattering induced by electronphonon interactions connects bands with a small energy bandwidth that would otherwise not carry any current in the ballistic limit of transport ${ }^{45,46}$. In ultra-short devices with bandstructure exhibiting several sub-bands with a low energy bandwidth, as $\langle 111\rangle$-oriented Si nanowires, the second effect dominates, thus leading to an increase of the current. However, in longer devices, backscattering plays the major role and the situation goes back to nor- 
TABLE II. Comparisons of 3 first LOA currents and corresponding Padé approximants $(0 / 1,1 / 1$, and 1/2) with the ballistic and SCBA currents in p-Si $\langle 100\rangle, \mathrm{p}-\mathrm{Si}\langle 110\rangle, \mathrm{p}-\mathrm{Si}\langle 111\rangle$, and p-Ge $\langle 110\rangle$ devices. Relative differences $(\varepsilon)$ of the LOA and Padé currents with respect to the SCBA values are also reported $\left(\varepsilon=100 \times\left|\mathcal{I}_{S C B A}-\mathcal{I}\right| / \mathcal{I}_{S C B A}\right)$ where $\mathcal{I}$ is ballistic, LOA, or Padé current.

\begin{tabular}{cccccccccc}
\hline \hline & Ballistic & LOA1 & LOA2 & LOA3 & Padé 0/1 & Padé 1/1 & Padé 1/2 & SCBA & Device \\
\hline Current [A] & $6.10 \mathrm{e}-6$ & $1.96 \mathrm{e}-6$ & $3.53 \mathrm{e}-8$ & $-3.36 \mathrm{e}-5$ & $3.63 \mathrm{e}-6$ & $-1.65 \mathrm{e}-6$ & $3.89 \mathrm{e}-6$ & $4.31 \mathrm{e}-6$ & $\mathrm{p}-\mathrm{Si}\langle 100\rangle$ \\
$\varepsilon[\%]$ & 41.5 & 54.5 & 99.2 & 879.6 & 15.8 & 138.3 & 9.7 & 0.0 \\
\hline Current [A] & $6.26 \mathrm{e}-6$ & $3.77 \mathrm{e}-6$ & $5.07 \mathrm{e}-6$ & $1.81 \mathrm{e}-5$ & $4.48 \mathrm{e}-6$ & $4.66 \mathrm{e}-6$ & $4.48 \mathrm{e}-6$ & $4.74 \mathrm{e}-6$ \\
$\varepsilon[\%]$ & 32.1 & 20.5 & 7.0 & 281.9 & 5.5 & 1.7 & 5.5 & 0.0 & $\mathrm{p}-\mathrm{Si}\langle 110\rangle$ \\
\hline Current [A] & $5.29 \mathrm{e}-6$ & $8.18 \mathrm{e}-6$ & $-4.12 \mathrm{e}-5$ & $3.70 \mathrm{e}-3$ & $1.16 \mathrm{e}-5$ & $5.45 \mathrm{e}-6$ & $9.08 \mathrm{e}-6$ & $4.85 \mathrm{e}-6$ & $\mathrm{p}-\mathrm{Si}\langle 111\rangle$ \\
$\varepsilon[\%]$ & 9.1 & 68.7 & 949.5 & 76188.7 & 139.2 & 12.4 & 87.2 & 0.0 & \\
\hline Current [A] & $5.80 \mathrm{e}-6$ & $4.11 \mathrm{e}-6$ & $4.70 \mathrm{e}-6$ & $5.03 \mathrm{e}-7$ & $4.49 \mathrm{e}-6$ & $4.55 \mathrm{e}-6$ & $4.49 \mathrm{e}-6$ & $4.74 \mathrm{e}-6$ & $\mathrm{p}-\mathrm{Ge}\langle 110\rangle$ \\
$\varepsilon[\%]$ & 22.4 & 13.3 & 0.8 & 89.4 & 5.3 & 4.0 & 5.3 & 0.0 \\
\hline \hline
\end{tabular}

mal, i.e. the current magnitude decreases when electronphonon scattering is turned on. The case of p-type device is more pathological since the converged SCBA becomes smaller than the ballistic value. Such a configuration seems to involve more complex physical phenomena that require to consider higher order LOA currents. It clearly illustrates the limit of our technique to reproduce SCBA currents. We also mention that it has been recently shown ${ }^{47,48}$ that the SCBA with Luttinger-Ward Functional self-energy may produce unphysical convergence for strong-coupling system. $\langle 111\rangle$ p-type direction could be one of these configurations. We can also note that a similar approach using Hypergeometric resummation has been recently proposed and could be tested in such case ${ }^{49}$. However, we suggest to take as a warning signal of the accuracy of the present approach the situation where first order LOA current is larger than the ballistic one.

In summary we have proposed a numerically efficient technique to reproduce the SCBA currents. It relies on the LOA coupled to Padé approximants and only requires the first few iterations of the SCBA procedure. The method has been applied to NW transistors using an atomistic 3D quantum transport code. We focused on the saturation regime current of the devices at high gate bias and compared the LOA and Padé currents with the SCBA values. In particular, we showed that the Padé $1 / 2$ with only first three order LOA currents can successfully reproduce the SCBA currents with a small relative difference $(<10 \%)$ in most of considered devices except for p-Si $\langle 111\rangle$ device where the electronphonon interaction exhibits singular effects. We also suggested as a warning signal to SCBA solution the case where first order LOA current is larger than ballistic one.

This work was granted access to the HPC resources of Aix-Marseille Université financed by the project Equip@Meso (ANR-10-EQPX-29-01) and funded by the NOODLES contract (ANR-13-NANO-0009) of the ANRFrench National Research Agency. The author Y. Lee would like to thank the AMIDEX for financial support.
* youseung.lee@im2np.fr

† marc.bescond@im2np.fr

1 Y. Lee, M. Lannoo, N. Cavassilas, M. Luisier, and M. Bescond, Phys. Rev. B 93, 205411 (2016).

2 "Intel 22nm 3-d tri-gate transistor technology," (2011).

${ }^{3}$ H. Mertens et al., in Symp. VLSI Technol. (2016) pp. 1-2.

${ }^{4}$ L. Li, Y. Yu, G. J. Ye, Q. Ge, X. Ou, H. Wu, D. Feng, X. H. Chen, and Y. Zhang, Nat. Nanotech. 9, 372 (2014).

5 A. D. Franklin, M. Luisier, S.-J. Han, G. Tulevski, C. M. Breslin, L. Gignac, M. S. Lundstrom, and W. Haensch, Nano Lett. 12, 758 (2012).

${ }^{6}$ B. Radisavljevic, A. Radenovic, J. Brivio, V. Giacometti, and A. Kis, Nat. Nanotech. 6, 147 (2011).

7 Y. Yoon, K. Ganapathi, and S. Salahuddin, Nano Lett. 11, 3768 (2011).

8 A. Szabó, R. Rhyner, and M. Luisier, Phys. Rev. B 92 , 035435 (2015).

9 J. Cao, D. Logoteta, S. Özkaya, B. Biel, A. Cresti, M. G. Pala, and D. Esseni, IEEE Trans. Electron Devices PP,
$1(2016)$.

10 K. Tomioka, M. Yoshimura, and T. Fukui, Nature 488, 189 (2012).

11 L.-F. Shen, S. Yip, Z. xing Yang, M. Fang, T. Hung, E. Y. Pun, and J. C. Ho, Sci. Rep. 5 (2015).

12 X. Zhou, Symp. VLSI Technol. (2016).

13 M. Di Ventra, S. T. Pantelides, and N. D. Lang, Phys. Rev. Lett. 84, 979 (2000).

14 S. Poli, M. G. Pala, T. Poiroux, S. Deleonibus, and G. Baccarani, IEEE Trans. Electron Devices 55, 2968 (2008).

15 H. Carrillo-Nuñez, M. Bescond, N. Cavassilas, E. Dib, and M. Lannoo, J. Appl. Phys. 116, 164505 (2014).

16 H. M. Yann-Michel Niquet and C. Delerue, Appl. Phys. Lett. 100, 153119 (2012).

17 M. Kobayashi and T. Hiramoto, J. Appl. Phys. 103, 053709 (2008).

18 X. Zhao, C. M. Wei, L. Yang, and M. Y. Chou, Phys. Rev. Lett. 92, 236805 (2004).

19 G. Pizzi, M. Gibertini, E. Dib, N. Marzari, G. Iannaccone, 
and G. Fiori, Nat. Commun. 7 (2016).

20 M. Moussavou, N. Cavassilas, E. Dib, and M. Bescond, J. Appl. Phys. 118, 114503 (2015).

21 A. Lherbier, M. P. Persson, Y.-M. Niquet, F. Triozon, and S. Roche, Phys. Rev. B 77, 085301 (2008).

${ }^{22}$ M. Luisier and G. Klimeck, Phys. Rev. B 80, 155430 (2009).

23 F. Murphy-Armando, G. Fagas, and J. C. Greer, Nano Lett. 10, 869 (2010).

24 S. Datta, Electronic Transport in Mesoscopic Systems (Cambridge University Press, Cambridge, U.K., 1995).

25 D. K. Ferry and S. M. Goodnick, Transport in Nanostructures (Cambridge University Press, Cambridge, U.K., 1997).

26 H. Haug and A.-P. Jauho, Quantum Kinetics in Transport and Optics of Semiconductors, vol. 123 of Springer Series in Solid-State Sciences (Springer, Berlin, New York, 1996).

27 A. Svizhenko, M. P. Anantram, T. R. Govindan, B. Biegel, and R. Venugopal, J. Appl. Phys. 91, 2343 (2002).

28 S. Jin, Y. J. Park, and H. S. Min, J. Appl. phys. 99, 123719 (2006).

${ }^{29}$ G. Mil'nikov and N. Mori, J. Comput. Electron. 15, 1179 (2016).

30 R. Rhyner and M. Luisier, Phys. Rev. B 89, 235311 (2014).

31 R. Rhyner and M. Luisier, Nano Lett. 16, 1022 (2016).

32 H. Mera, M. Lannoo, C. Li, N. Cavassilas, and M. Bescond, Phys. Rev. B 86, 161404 (2012).

${ }^{33}$ H. Mera, M. Lannoo, N. Cavassilas, and M. Bescond, Phys. Rev. B 88, 075147 (2013).
34 G. H. Hardy, Divergent Series (Chelsea, New York, 1991).

35 J. G. A. Baker and P. Graves-Morris, Padé Approximants (Cambridge University Press, Cambridge, 1996).

36 J. C. Slater and G. F. Koster, Phys. Rev. 94, 1498 (1954).

37 T. B. Boykin, G. Klimeck, and F. Oyafuso, Phys. Rev. B 69, 115201 (2004).

38 S. Lee, F. Oyafuso, P. von Allmen, and G. Klimeck, Phys. Rev. B 69, 045316 (2004).

39 Z. Sui and I. P. Herman, Phys. Rev. B 48, 17938 (1993).

40 A. Paul, M. Luisier, and G. Klimeck, J. Comput. Electron. 9, 160 (2010).

41 T. Kubis and P. Vogl, J. Comput. Electron. 6, 183 (2007).

42 N. Cavassilas, F. Michelini, and M. Bescond, J. Comput. Electron. 15, 1233 (2016).

43 R. Rhyner and M. Luisier, J. Appl. phys. 114, 223708 (2013)

44 G. D. Mahan, Many-Particle Physics (Plenum, New York, 1990).

45 J. Chang, L. F. Register, and S. K. Banerjee, Appl. Phys. Lett. 103, 223509 (2013).

46 A. Szabó, Dissipative quantum transport simulations in two-dimensional semiconductor devices from first principles, Ph.D. thesis, ETH Zurich (2016).

47 W. Lee, N. Jean, and S. Sanvito, Phys. Rev. B 79, 085120 (2009).

48 A. Stan, P. Romaniello, S. Rigamonti, L. Reining, and J. A. Berger, New J. Phys 17, 093045 (2015).

49 H. Mera, T. G. Pedersen, and B. K. Nikolić, Phys. Rev. B 94, 165429 (2016). 